WellBeing International

WBI Studies Repository

$1-2017$

\title{
Individual Personality Differences in Goats Predict Their Performance in Visual Learning and Non-Associative Cognitive
} Tasks

\author{
Christian Nawroth \\ Queen Mary University of London \\ Pamela M. Prentice \\ University of Edinburgh \\ Alan G. McElligott \\ Queen Mary University of London
}

Follow this and additional works at: https://www.wellbeingintlstudiesrepository.org/leaabi

Part of the Animal Studies Commons, Comparative Psychology Commons, and the Other Animal Sciences Commons

\section{Recommended Citation}

Nawroth, C., Prentice, P. M., \& McElligott, A. G. (2017). Individual personality differences in goats predict their performance in visual learning and non-associative cognitive tasks. Behavioural processes, 134, 43-53.

This material is brought to you for free and open access by WellBeing International. It has been accepted for inclusion by an authorized administrator of the WBI Studies Repository. For more information, please contact wbisr-info@wellbeingintl.org.

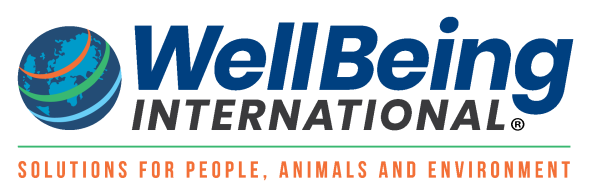


1 Reference: Nawroth, Prentice, McElligott (2016) Individual personality differences in

2 goats predict their performance in visual learning and non-associative cognitive tasks.

3 Behavioural Processes, available online. doi: 10.1016/i.beproc.2016.08.001

4

$5 \quad$ Link to published article

6

7

8 Individual personality differences in goats predict their performance in visual learning

9 and non-associative cognitive tasks

10

11 Christian Nawroth ${ }^{\star 1}$, Pamela M. Prentice ${ }^{\star^{2}}$, Alan G. McElligott ${ }^{1}$

121 Queen Mary University of London, Biological and Experimental Psychology, School of 13 Biological and Chemical Sciences, London, UK

$14{ }^{2}$ University of Edinburgh, Royal (Dick) School of Veterinary Studies, Edinburgh, UK

* both authors contributed equally to this work

Corresponding authors

Christian Nawroth

Email address: nawroth.christian@gmail.com

Alan G. McElligott

27 Email address: a.g.mcelligott@qmul.ac.uk 
29

30 - We investigated goat personality and performance in different cognitive tasks

31 - Feature cue preference of goats was also examined

- Slow explorers performed better in a non-associative cognitive task

33 - Less sociable subjects performed better in a visual discrimination task - Good visual learning performance was associated with a preference for feature cues 35 
39 Variation in common personality traits, such as boldness or exploration, is often associated with 40 risk-reward trade-offs and behavioural flexibility. To date, only a few studies have examined 41 the effects of consistent behavioural traits on both learning and cognition. We investigated 42 whether certain personality traits ('exploration' and 'sociability') of individuals were related to 43 cognitive performance, learning flexibility and learning style in a social ungulate species, the 44 goat (Capra hircus). We also investigated whether a preference for feature cues rather than 45 impaired learning abilities can explain performance variation in a visual discrimination task. We 46 found that personality scores were consistent across time and context. Less explorative goats 47 performed better in a non-associative cognitive task, in which subjects had to follow the trajectory of a hidden object (i.e. testing their ability for object permanence). We also found that 49 less sociable subjects performed better compared to more sociable goats in a visual discrimination task. Good visual learning performance was associated with a preference for

51 feature cues, indicating personality-dependent learning strategies in goats. Our results suggest 52 that personality traits predict the outcome in visual discrimination and non-associative cognitive 53 tasks in goats and that impaired performance in a visual discrimination tasks does not 54 necessarily imply impaired learning capacities, but rather can be explained by a varying 55 preference for feature cues.

57 Keywords: cognition; exploration; feature cues; learning type; sociability; transposition 


\section{Introduction}

Personalities refer to consistent individual differences in behaviour across time and context (Réale et al., 2007), and have been demonstrated across many taxa (Briard et al., 2015; David et al., 2011; Morton et al., 2015; Pike et al., 2008; Rodríguez-Prieto et al., 2011). Consistent behavioural responses are traditionally arranged along five key personality axes that include exploration/avoidance, bold/shy, aggression, sociability and activity (Gosling, 2001; Réale et al., 2007). Accumulating evidence suggests that the variation of personality traits observed within populations is heritable and has fitness advantages (Dingemanse et al., 2002; Sih et al., 2004). Personality traits are therefore likely to be under evolutionary selection.

According to selection theory, the maintenance of variation in personality traits within a population results from individual trade-offs between current and future fitness returns (Dall et al., 2004; Wolf et al., 2007). Individuals directing energy towards immediate fitness goals will display risky behaviours, such as boldness toward predators or fast explorers of novel situations (Sih et al., 2004). In contrast, individuals concentrating energy towards future fitness goals will display risk-averse behaviours, displaying shy and non-aggressive behaviours towards other individuals and be slow to explore novel environments.

Consistency in risk prone or risk aversive behaviours within a population (otherwise known as coping strategies) are made up of suites of correlated personality traits (Drent et al., 2003; Verbeek et al., 1996). Correlations between boldness, aggression and exploration have been seen in many taxa and reflect alternatives in life history strategies (Dingemanse et al., 2004; Marchetti and Drent, 2000; Wolf et al., 2007). If correlations between personality traits are components of broader life-history strategies, then it might be expected that individual variation in other behavioural and physiological traits could also be related and exist within populations. It has been suggested that personality trait differences (e.g. bold, fast explorers or shy, slow explorers) may be linked to variation in behavioural flexibility and learning performance via differences in attention to environmental cues and the degree to which internal and external 
cues guide behaviour (Carere and Locurto, 2011). For example, in great tits (Parsus major), slow explorers respond to external stimuli by readily adjusting their behaviour to changes in their environment, compared to fast explorers that have active routines and do not perform well in fluctuating environments (Verbeek et al., 1996). It could therefore be expected that slow explorers would excel in acquiring information in situations when changes in environmental cues are relevant, such as locations or stimuli associated with food (Guillette et al., 2011). Such differences in internal and external cues for guiding behaviour can then be the link by which personality affects behavioural responsiveness, learning ability and successful adjustment to environmental changes.

The relationship between personality traits and learning ability is also supported by several studies on reversal learning. In reversal learning, animals first learn to discriminate between two stimuli, where one of the stimuli is rewarded. In the reversal phase, the rewarded stimulus is changed; the previously unrewarded stimulus now becomes rewarded, requiring a shift of attention. Fast explorers (or bold and proactive animals) are better at learning an initial discrimination task (Benus et al., 1987; Guillette et al., 2009) but slow explorers (or shy, reactive animals) are better at adapting to changes in an already learned task (Bolhuis et al., 2004; Guillette et al., 2011). However, some studies in birds do not show this relationship between explorative behaviour and behavioural flexibility (Bebus et al., 2016; Cole et al., 2011), while others even show the opposite effect (Bousquet et al., 2015).

Sociability (a key axis of animal personality) is defined by Réale et al. (2007) as an individual's reaction to the presence or absence of conspecifics. Like explorative behaviour, it is also connected to other behavioural traits, particularly in the social domain. For example, juvenile dogs (Canis familiaris) that are highly sociable are more likely to adopt an interactive conflict resolution strategy, while those that are less sociable tend to react passively (Riemer et al., 2013). Shy female kangaroos (Macropus giganteus) have fewer preferred associates within populations, and also have significantly larger mean foraging group sizes compared to bolder individuals, indicating a link between boldness and sociability of subjects (Best et al., 2015). 
116 Therefore, although exploration and sociability can be interconnected (e.g. Best et al., 2015),

117 little is known how sociability is related to behavioural flexibility and learning performance.

119 Only a few studies (predominantly on primates) have investigated how consistent behavioural 120 traits, such as boldness or sociability, predict performance in non-associative cognitive tasks. 121 For example, common marmosets (Callithrix jacchus) that express higher emotional reactivity 122 show a lower participation rate in a cognitive task in which they had to use human-given cues to 123 find food (Schubiger et al., 2015). However, the level of emotional reactivity did not predict 124 performance in the task. Other research rated capuchin monkeys (Cebus apella) on five personality dimensions and analyzed their participation rate and performance in a specific cognitive task (Morton et al., 2013). In this task, a small opaque cup was placed in front of one compartment and a larger opaque cup in front of another compartment. The correct response was to sit in the compartment that had the larger cup in front of it. Individuals that scored high on openness were more likely to participate in the task, whereas less assertive subjects performed better in the task. This negative effect of assertiveness may be best understood as individuals having motivational priorities other than food.

For ungulates, most studies investigating behavioural types and correlates to other behavioural traits focused on coping types (being either proactive or reactive). Pig (Sus scrofa) coping types are associated with aggression (Melotti et al., 2011) and also affect exploration patterns but not performance in a maze task (Jansen et al., 2009). In goats, Lyons (1989) found that they express consistent individual differences in temperament, in terms of reaction to humans (timidity), which reliably predict differences in pituitary adrenal responsiveness. Additionally, social strategies in conflict resolution is associated with personality traits in goats (Miranda-de la Lama et al., 2011). Recently, it was shown that personality traits such as exploration/avoidance behaviour and sociability towards other goats are stable over a time interval of about one year.

142 In addition, heart rate responses of the goats were predicted by their sociability outcomes, but not their exploration/avoidance behaviour (Briefer et al., 2015). 
145 We examined whether personality traits predict performance in learning and non-associative 146 cognitive tasks in goats. We first assessed inter-individual differences in exploration behaviour 147 towards novel items and altered environments, and sociability towards other goats by using four 148 different behavioural tests. 'Exploration' scores were obtained using an altered environment and 149 a novel object task (Dingemanse et al., 2007; Lansade et al., 2008a). 'Sociability' was 150 measured using behavioural expressions during the presence and absence of conspecifics 151 (Lansade et al., 2008b). We combined all the correlated behaviours in one score for each 152 personality trait, i.e. exploration and sociability (McGregor, 2013). Goats then participated in 153 three tasks. In a visual discrimination task, we investigated the ability of goats to associate a 154 reward with the colour of a particular container. In a non-associative cognitive task, goats had to 155 track the movement of two containers that crossed paths; a so called transposition task that has 156 previously been successfully tested on goats (Nawroth et al., 2015). Finally, we determined the 157 affinity of goats towards feature cues (such as shape and colour) by providing them with a brief experience of combined feature and spatial cues during a short training period. In the test,

159 feature and spatial cues were incongruent and by analyzing the goats' choices, we could infer 160 their inclination to orient on feature rather than spatial cues. We expected fast explorers to 161 perform better in the visual discrimination task learning (Guenther et al., 2014; Guillette et al., 162 2009). In addition, we predicted that performance in the visual discrimination task and a non163 associative cognitive task would be predicted by the sociability of the test subjects, with higher 164 sociability scores resulting in poorer performance due to differences in motivation rather than on 165 different cognitive skills (Morton et al., 2013). If poor performance in a visual discrimination task 166 was not caused by impaired learning abilities but rather due to a different learning strategy, we 167 expected that better visual learners would show higher affinity for feature cues in the preference 168 task. 


\subsection{Ethics statement}

172

173 Animal care and all experimental procedures were in accordance with the ASAB/ABS

174 Guidelines for the Use of Animals in Research (Association for the Study of Animal Behaviour, 175 2016). The study was approved by the Animal Welfare and Ethical Review Board of Queen 176 Mary University of London. All measurements were non-invasive, and the experiment lasted not 177 more than 10 min for each individual goat. If the goats had become stressed, the test would have been stopped.

\subsection{Animals, keeping and management}

The study was carried out at Buttercups Sanctuary for Goats (http://www.buttercups.org.uk), UK. We tested 16 adult goats (eight females and eight castrated males), which were fully habituated to human presence due to previous research (Baciadonna et al., 2013; Briefer and McElligott, 2013). They were between 2-11 years of age and of various breeds (Table 1). Routine care of the animals was provided by sanctuary employees and volunteers. The goats had ad libitum access to hay and were not food restricted before testing. The experiments were carried out in temporary enclosures, within the normal daytime range of the goats. Subjects were tested from 12:00-16:00h from May to July 2015.

\subsection{Personality tests}

192

193 Two personality traits relevant to social species were measured: Exploration (i.e. reaction to a new situation) and sociability (i.e. reaction to the presence or absence of conspecifics (Réale et al., 2007)). Two separate experiments were conducted per trait to test for consistency across situations. Additionally, each personality test was repeated at two different time points (3 week intervals) to test for consistency over time. Exploration was measured using a novel object test 
198 (Lansade et al., 2008a) and an altered environment test (Dingemanse et al., 2007), both in

199 familiar environments. Sociability was measured using an attraction test in a familiar

200 environment and an isolation test in a less familiar environment (Lansade et al., 2008b). Several

201 behavioural responses were measured from each test (Table A.1 in Appendix). A principal

202 component analysis (PCA) was carried out in order to combine all correlated behaviours into

203 one score, which would be representative of the response of the subjects to the tests

204 (McGregor, 2013). From the resulting PCA (one for each personality trait), we selected the

205 scores of the most relevant principal component (PC), verified score consistency over the three

206 weeks, and averaged the PC scores to obtain one personality score for each goat. The

207 personality tests and methods used to calculate personality scores are detailed in the Appendix,

208 and the resulting scores for 15 goats tested in this study are listed in Table 1. Note that one

209 subject had to be excluded due to a consistent side bias in the tests described below (see

210 results section). Highly positive exploration scores indicated goats that spent more time

211 exploring the novel item or environment. Highly positive sociability scores indicated highly social

212 goats (i.e. reacted strongly to isolation and spent time with other goats during the attraction test;

213 see Appendix).

214

215 Table 1 Information on test subjects: ID, breed, sex, age and personality scores (exploration 216 and sociability).

\begin{tabular}{lllrrr}
\hline Goat & Breed & Sex & Age in years & Exploration & Sociability \\
\hline 1 & Boer & female & 2 & 2.90 & 2.67 \\
2 & Anglo Nubian & female & 7 & -0.71 & 1.78 \\
3 & Anglo Nubian & male & 4 & -1.85 & 0.14 \\
4 & Anglo Nubian & male & 4 & -3.18 & -1.44 \\
5 & Anglo Nubian & male & 6 & -1.22 & -1.90 \\
6 & Mix & female & 5 & 1.94 & 1.24 \\
7 & Mix & male & 7 & -0.17 & -2.25 \\
8 & Anglo Nubian & female & 10 & -0.53 & 1.29 \\
9 & British Saanen & male & 7 & 0.04 & -1.31 \\
10 & British Alpine & female & 11 & 2.41 & -1.41 \\
11 & Anglo Nubian & female & 9 & -3.85 & -2.28 \\
12 & British Toggenburg & male & 5 & 1.24 & 0.13 \\
13 & British Toggenburg & male & 7 & 0.58 & -3.05
\end{tabular}


221 For training and testing in the visual discrimination task and the non-associative cognitive task, 222 individual goats were physically and visually separated from conspecifics in a test pen $(2 \mathrm{~m} \mathrm{x}$ $2232.5 \mathrm{~m}$ ). An experimenter positioned himself in an adjacent compartment separated from the 224 tested subject by grating, allowing subjects to insert their snouts through two openings positioned on either the left or right side of the grating $(12 \mathrm{~cm} \times 10 \mathrm{~cm})$. The distance between the openings was $30 \mathrm{~cm}$. A sliding board $(58 \mathrm{~cm} \times 30 \mathrm{~cm}$ ) was placed on a small table (height: $45 \mathrm{~cm}$ ) in front of the grating (Figure 1a). For the feature preference task, goats were physically and visually separated from conspecifics in a larger test pen $(3 \times 6 \mathrm{~m})$. Subjects received a habituation trial prior to training and testing to determine whether they displayed any signs of stress during isolation. Half of the subjects received the visual discrimination task first, while the other half received the cognitive task first. The feature preference task was always administered last.

\subsubsection{Visual discrimination task}

Goats were assigned to an initial training period of four trials in total. In each trial, the experimenter either placed a black or a white cup (diameter: $9 \mathrm{~cm}$; height: $13 \mathrm{~cm}$ ) at the left or right side of the board and baited it in full view of the subject with a piece of pasta. The subject then had the opportunity to make a choice by putting their snout through one of the outer left or right gaps in the grating and, if chosen correctly, obtained the reward (Figure 1a). In the discrimination task, half of the subjects received a reward by choosing the black cup while the other subjects received a reward by choosing the white cup. Before each test trial, the experimenter baited the corresponding cup surreptitiously. Each different coloured cup was then either placed on the left or right hand side of the sliding board at a distance of about $35 \mathrm{~cm}$. After approximately $2 \mathrm{~s}$, the experimenter pushed the board towards the grating. The subject made its choice by putting its snout through one of the left or right gaps in the grating and if 
learning criterion when they achieved eight correct choices in eight consecutive trials ( $8 / 8$ would result in $P<0.008$; binomial test). They received a total number of three sessions, each consisting of 32 trials. Maximum trial number was set at 96 trials (supplementary video).

\subsubsection{Non-associative cognitive task}

In four training trials prior to testing, the experimenter placed a brown cup (diameter: $9 \mathrm{~cm}$; height: $11 \mathrm{~cm}$ ) on either the left or right hand side of the board. The cup was baited in full view of the subject with a small reward (a piece of dry pasta). Immediately afterwards, the experimenter pushed the board towards the grating and the subject was allowed to make a choice by placing its snout through the appropriate gap. In test trials, subjects were presented with a transposition in which two identical brown cups crossed paths and their positions switched. Two cups were placed on the left and the right side of the sliding board and a food reward was placed in one of the cups in full view of the subject. After baiting, the experimenter simultaneously moved the left cup to the right side, and the right cup to the left side of the board, so that the cups crossed path in the middle. The baited cup crossed path in the direction of the test subject in $50 \%$ of the trials. After the transposition, the experimenter immediately pushed the sliding board towards the grating, allowing the subjects to make a choice. Each subject received only one test session of 12 trials (supplementary video).

\subsubsection{Feature preference task}

The feature preference task was conducted one week after subjects participated in the visual discrimination task and the cognitive task. Two different types of containers were used: a square brown container (length: $16 \mathrm{~cm}$, height: $18 \mathrm{~cm}$ ) and a round black container (diameter: $14 \mathrm{~cm}$, height: $9 \mathrm{~cm}$ ). In all trials, three containers were positioned in total in the test area. In training trials, either one rewarded round black container, or one rewarded square brown container was positioned at the left or right side of a semicircle, respectively. Two additional containers of the other respective type were positioned in the middle and on the opposite side of 
277 the semicircle (see Figure 1b) but remained unrewarded. The containers were arranged in a 278 semicircle so that each container had the same distance towards the starting point of the goat $279(250 \mathrm{~cm})$. The distance between containers was $50 \mathrm{~cm}$. Each subject received three training 280 trials. In these trials, an experimenter baited either the left or right container (either the single 281 square brown container or the single round black container) in full view of the subject whilst they approached the experimenter. In all trials, the subject moved directly towards the baited location. After training, subjects proceeded to the preference trials. During the preference test, all containers were baited with a food reward and the position of the former rewarded container was changed from the right to left position or vice versa, depending on the former experienced location. After a trial had finished, the experimenter re-baited the container chosen by the goat and sham-baited the other two containers. Each subject received 10 successive preference trials. Seven subjects started with the rewarded container positioned on the left side, while 8 subjects started with the rewarded container on the right side. For eight subjects, the rewarded container was the square brown container, for seven subjects it was the round black container.

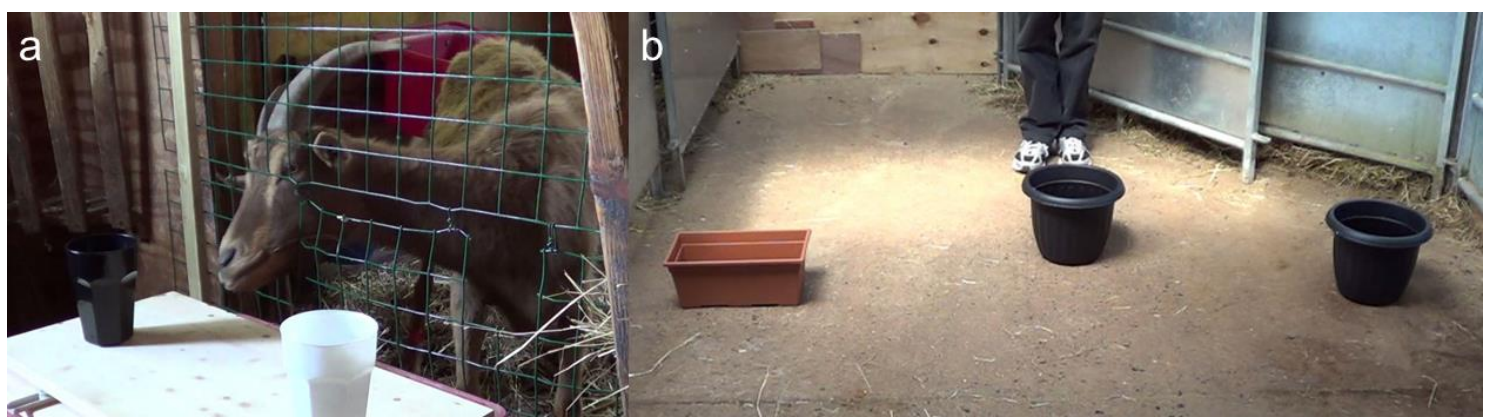

293 Figure 1 Setup of the experiments: a) visual discrimination task (similar to the non-associative cognitive task) b) feature preference task

\subsection{Data scoring and analysis}

All trials were scored live during the test and were also videotaped (Sony HCR-CX190E Camcorder). A test trial ended after a goat made a choice or after $60 \mathrm{~s}$ had passed. For the visual discrimination $(N=12)$ and the cognitive task $(N=15)$, we scored the correct choices of all subjects. For the visual discrimination task, we ranked subjects based on the number of trials 
they needed to reach the learning criteria (lower rank numbers indicate faster learning) as trials were skewed towards the minimum (8) and the maximum amount of trial (96 trials) needed. In the feature preference task $(\mathrm{N}=15)$, we scored the total number of feature choices of subjects. A feature choice was defined as choosing the previously rewarded container (square brown or round black container) in a new spatial configuration. One subject had to be excluded from the analysis, because it consistently showed a side bias in all tasks. In the visual discrimination task, two subjects had to be excluded as they lost interest in the task quickly, and an additional subject was excluded because it was interfering with the grating. We used a linear regression model with step-wise elimination of non-significant parameters to determine whether the outcomes of the different tasks were predicted by their personality scores (exploration and sociability), including sex, age and breed as covariates. We then used Spearman rank correlations with a Bonferroni adjustment of the alpha level $(\alpha=0.017)$ to compare performances pairwise between the visual discrimination, cognitive, and feature preference task. All analyses were conducted in SPSS $\vee$ 22.0. a was set at 0.05 , unless specified otherwise.

\section{Results}

Subjects' sociability scores predicted their ranked learning performance, with less sociable subjects requiring fewer trials to reach the criterion $\left(R^{2}=0.337, F_{1,11}=5.09, P=0.048\right.$, Figure 2). Sociability scores also predicted the number of feature choices made by individuals in the feature preference task, with less sociable subjects choosing the feature container more often compared to more sociable ones $\left(R^{2}=0.418, F_{1,11}=5.09, P=0.009\right)$. Exploration scores predicted the performance in the cognitive task, with low explorative subjects performing better compared to highly explorative goats $\left(R^{2}=0.268, F_{1,14}=4.758, P=0.048\right.$; Figure 2$)$. Sex, age and breed had no significant effects on task outcomes. Finally, we calculated correlations between the performances of subjects in all three tasks. Good performance in the visual discrimination task was highly correlated with preference for feature cues $(r=-.608, \mathrm{~N}=12, P=$ 
330 0.009). No other significant correlations were found (Visual discrimination task $\mathrm{x}$ cognitive task: $r$

$331=-.016, \mathrm{~N}=12, P=0.94$; feature preference task $\mathrm{x}$ cognitive task: $r=0.087, \mathrm{~N}=15, P=0.68$ ).

332

333
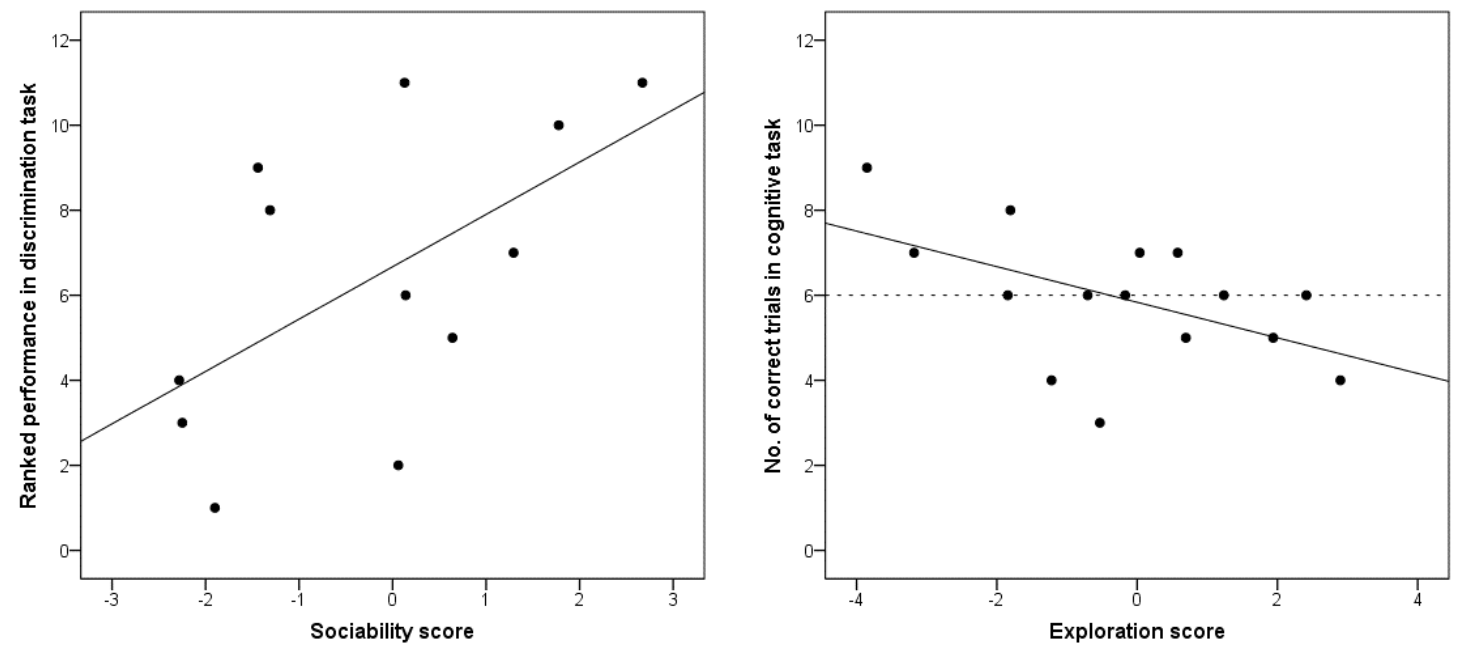

334 Figure 2 Relationship between the ranked performance in the visual discrimination task and the

335 PCA score for sociability (left) and the performance in the cognitive task and the PCA score for exploration (right); the dashed line indicates performance by chance level 


\section{Discussion}

339

340 We investigated how personality types in goats predicted the outcomes of various cognitive

341 tasks. Personality scores of individual subjects were repeatable over a short time period (3 342 weeks) and thus confirm previous findings (Briefer et al., 2015). Less explorative goats 343 performed better in a non-associative cognitive task, in which subjects had to follow the 344 trajectory of a hidden object. Performance in a visual discrimination task was related to their 345 sociability scores, with less sociable subjects outperforming more sociable ones. In addition, the 346 preference of subjects to relocate food patches using feature or other cues (as displayed in the 347 feature preference task) was predicted by personality scores. A high preference for feature cues 348 was associated with low sociability scores and good performance in the visual discrimination 349 task. Our results suggest that the personality profile of goats is related to their performance in various cognitive tasks (Guillette et al., 2009; Morton et al., 2013).

351

352 We found that exploration scores of subjects predicted their performance in a transposition task. 353 In this task, subjects had to keep track of a hidden object while two possible baiting containers 354 had their positions swapped. It is possible that even a short moment of distraction can lead to errors, because subjects may mistake the baited container with the empty one due to their identical appearance. Indeed, it has been shown that using two differently coloured and shaped boxes slightly improved performance in the task in goats (Nawroth et al., 2015). Thus, the good performance of less explorative goats in this transposition task may be explained by their potentially decreased arousal by additional external cues, leading to a higher inclination to pay attention to the task. Alternatively, it has been suggested that slow explorers are expected to perform better when changes in environmental cues are relevant, such as changes in locations or stimuli associated with food (Guillette et al., 2011, 2009). To date, only a few studies have investigated how personality traits predict outcomes and participation rate in non-associative cognitive tasks. Participation rate of common marmosets in an object choice task was related to their emotional reactivity (Schubiger et al., 2015), while capuchin monkeys with higher openness scores were more inclined to participate in a choice task (Morton et al., 2013). In light 
367

368

369

370

371

372

373

374

375

376

377

378

379

380

381

382

383

384

385

386

387

388

389

390

391

392

393

394

395

of these findings, our results may be best understood as highly explorative individuals having motivational priorities other than food rather than cognitive impairments. Controversially, there was no connection between goats' performance in a discrimination task and their exploration scores.

Previous research has found a correlation between learning performance and explorative behaviour (Guillette et al., 2011, 2009). Contrary to expectations, we did not find a similar effect. However, previous research has also shown mixed results. For example, when presented with a visual discrimination task and a reversal learning task, explorative behaviour was not linked to performance in Florida scrub-jays (Aphelocoma coerulescens) (Bebus et al., 2016). Performance in a visual discrimination task in our experiment was associated with sociability scores of our subjects, with less sociable subjects outperforming more sociable ones. More social subjects might by more stressed while tested individually, leading to distraction and poorer performance. This, in turn, may have resulted in decreased attention towards the task and longer time to reach the learning criterion. If this was the case, we might expect a similar effect for the non-associative cognitive task, but the performance in that task was not related to their sociability scores.

Interestingly, sociability was associated with a higher inclination to choose feature cues in a preference task. These results tend to point in the direction that sociability, or a certain personality type in general, can be linked to learning style, i.e. paying more attention to feature or spatial cues. This means that impaired learning capacities do not necessarily have to be the exclusive reason for poor performance in a visual discrimination task. In addition, other factors such as stress or arousal may contribute to a decline in performance, different preferences for learning of either feature and/or spatial cues have to be taken into account. Because memorizing food patches is essential when making foraging decisions, different strategies to achieve this goal are available. For example, subjects can relocate food patches by memorizing feature cues such as size and colour, or by spatially representing the environment, using local or global landmarks (Shettleworth, 2010). Using feature cues such as colour or shape to identify 
food patches can increase foraging efficiency. However, feature cues may change over time and therefore animals should use spatial cues to relocate food that occurs in a temporally stable position. For example, European greenfinch (Carduelis chloris) show context-dependent preferences in that they are able to learn feature and spatial cue types, while spatial cues are favoured over feature cues only in temporally stable contexts (Herborn et al., 2011). Some subjects may develop a preference for one cue type over the other due to different ontogenetic factors and individual experience.

403

Ungulates have been shown to possess personality traits that are stable over time (Briefer et al., 2015; Graunke et al., 2013). Previous research in goats has linked these traits to hormonal (pituitary adrenal responsiveness: Lyons, 1989) and physiological parameters (heart rate response: Briefer et al., 2015). To our knowledge, our results show first evidence for a link between certain personality traits and discrimination learning and cognitive performance in goats. In pigs, their coping types affected motivation and exploration, but not performance in an initial maze task (Jansen et al., 2009). By contrast, proactive pigs have been found to be less successful in a reversal learning task than reactive pigs (Bolhuis et al., 2004). This is in line with research on other species (Guillette et al., 2009) and suggests that proactive (i.e. more explorative) pigs have a higher propensity to develop inflexible behaviour.

415 We conclude that different personality traits predict the outcome in visual discrimination and non-associative cognitive tasks in goats. In addition, we found that an impaired performance in

417 a visual discrimination tasks does not necessarily imply an impairment of learning in general, 418 but rather can be explained due to different preferences towards feature or spatial cues. The 419 particular link between sociability and its association with learning performance and preferences 420 for particular cue types seems to be a promising area for future research and needs to be taken under consideration when discussing potential impairments in learning capacities in future research in other ungulate species. These findings will lead to advances in animal welfare in the long term by providing important insights into individual differences in personality traits in

424 livestock and how they are related to behavioural outcomes in different contexts. 
426 Acknowledgments

427

428 We would like to thank Luigi Baciadonna for test assistance and Katherine Herborn for 429 statistical advice. We thank Robert Hitch and all the staff and volunteers of Buttercups 430 Sanctuary for Goats (www.buttercups.org.uk) for their excellent help and free access to the 431 animals.

432

$433 \quad$ Funding

434

435 This work was supported by a grant from the Deutsche Forschungsgemeinschaft (NA 1233/1-1)

436 to C.N., and a grant from the Farm Sanctuary 'Someone Project' to A.G.M. and C.N. 
Association for the Study of Animal Behaviour, 2016. Guidelines for the treatment of animals in behavioural research and teaching. Anim. Behav. 111, I-IX.

Baciadonna, L., McElligott, A.G., Briefer, E.F., 2013. Goats favour personal over social information in an experimental foraging task. PeerJ 1, e172. doi:10.7717/peerj.172

Bebus, S.E., Small, T.W., Jones, B.C., Elderbrock, E.K., Schoech, S.J., 2016. Associative learning is inversely related to reversal learning and varies with nestling corticosterone exposure. Anim. Behav. 111, 251-260. doi:10.1016/j.anbehav.2015.10.027

Benus, R.F., Koolhaas, J.M., van Oortmerssen, G.A., 1987. Individual differences in behavioural reaction to a changing environment in mice and rats. Behaviour 100, 105-121. doi:10.1163/156853987X00099

Best, E.C., Blomberg, S.P., Goldizen, A. W., 2015. Shy female kangaroos seek safety in numbers and have fewer preferred friendships. Behav. Ecol. 26, 639-646. doi:10.1093/beheco/arv003

Briefer, E.F., Oxley, J.A., McElligott, A.G., 2015. Autonomic nervous system reactivity in a freeranging mammal: effects of dominance rank and personality. Anim. Behav. 110, 121-132. doi:10.1016/j.anbehav.2015.09.022 
Carere, C., Locurto, C., 2011. Interaction between animal personality and animal cognition. Curr. Zool. 57, 491-498.

Carter, A.J., Feeney, W.E., Marshall, H.H., Cowlishaw, G., Heinsohn, R., 2013. Animal personality: what are behavioural ecologists measuring? Biol. Rev. 88, 465-475.

Cohen, S., Wills, T.A., 1985. Stress, social support, and the buffering hypothesis. Psychol. Bull. $98,310$.

Cole, E.F., Cram, D.L., Quinn, J.L., 2011. Individual variation in spontaneous problem-solving performance among wild great tits. Anim. Behav. 81, 491-498. doi:10.1016/j.anbehav.2010.11.025

Comrey, A.L., Lee, H.B., 2013. A first course in factor analysis. Psychology Press.

Dall, S.R.X., Houston, A.I., McNamara, J.M., 2004. The behavioural ecology of personality: consistent individual differences from an adaptive perspective. Ecol. Lett. 7, 734-739.

David, M., Auclair, Y., Cézilly, F., 2011. Personality predicts social dominance in female zebra finches, Taeniopygia guttata, in a feeding context. Anim. Behav. 81, 219-224.

Dingemanse, N.J., Both, C., Drent, P.J., Tinbergen, J.M., 2004. Fitness consequences of avian personalities in a fluctuating environment. Proc. R. Soc. Lon. B 271, 847-852.

Dingemanse, N.J., Both, C., Drent, P.J., Van Oers, K., Van Noordwijk, A.J., 2002. Repeatability and heritability of exploratory behaviour in great tits from the wild. Anim. Behav. 64, 929938.

Dingemanse, N.J., Wright, J., Kazem, A.J.N., Thomas, D.K., Hickling, R., Dawnay, N., 2007. Behavioural syndromes differ predictably between 12 populations of three-spined stickleback. J. Anim. Ecol. 76, 1128-1138. doi:10.1111/j.1365-2656.2007.01284.x

Drent, P.J., van Oers, K., van Noordwijk, A.J., 2003. Realized heritability of personalities in the great tit (Parus major). Proc. R. Soc. Lon. B 270, 45-51.

Gosling, S.D., 2001. From mice to men: what can we learn about personality from animal research? Psychol. Bull. 127, 45-86.

Graunke, K.L., Nürnberg, G., Repsilber, D., Puppe, B., Langbein, J., 2013. Describing temperament in an ungulate: a multidimensional approach. PLOS ONE 8, e74579. doi:10.1371/journal.pone.0074579 
Guenther, A., Brust, V., Dersen, M., Trillmich, F., 2014. Learning and personality types are related in cavies (Cavia aperea). J. Comp. Psychol. 128, 74-81. doi:10.1037/a0033678

Guillette, L.M., Reddon, A.R., Hoeschele, M., Sturdy, C.B., 2011. Sometimes slower is better: slow-exploring birds are more sensitive to changes in a vocal discrimination task. Proc. Biol. Sci. 278, 767-773. doi:10.1098/rspb.2010.1669

Guillette, L.M., Reddon, A.R., Hurd, P.L., Sturdy, C.B., 2009. Exploration of a novel space is associated with individual differences in learning speed in black-capped chickadees, Poecile atricapillus. Behav. Proc. 82, 265-270. doi:10.1016/j.beproc.2009.07.005

Hänninen, L., Pastell, M., 2009. CowLog: Open-source software for coding behaviors from digital video. Behav. Res. Methods 41, 472-476.

Herborn, K., Alexander, L., Arnold, K.E., 2011. Colour cues or spatial cues? Context-dependent preferences in the European greenfinch (Carduelis chloris). Anim. Cogn. 14, 269-277. doi:10.1007/s10071-010-0360-7

Jansen, J., Bolhuis, J.E., Schouten, W.P., Spruijt, B., Wiegant, V., 2009. Spatial learning in pigs: effects of environmental enrichment and individual characteristics on behaviour and performance. Anim. Cogn. 12, 303-315. doi:10.1007/s10071-008-0191-y

Lansade, L., Bouissou, M.-F., Erhard, H.W., 2008a. Fearfulness in horses: A temperament trait stable across time and situations. Appl. Anim. Behav. Sci. 115, 182-200.

Lansade, L., Bouissou, M.-F., Erhard, H.W., 2008b. Reactivity to isolation and association with conspecifics: a temperament trait stable across time and situations. Appl. Anim. Behav. Sci. 109, 355-373.

Lyons, D.M., 1989. Individual differences in temperament of dairy goats and the inhibition of milk ejection. Appl. Anim. Behav. Sci. 22, 269-282. doi:10.1016/0168-1591(89)90022-1

Marchetti, C., Drent, P.J., 2000. Individual differences in the use of social information in foraging by captive great tits. Anim. Behav. 60, 131-140.

McGregor, P.K., 2013. Playback and studies of animal communication. Springer Science \& Business Media.

Melotti, L., Oostindjer, M., Bolhuis, J.E., Held, S., Mendl, M., 2011. Coping personality type and environmental enrichment affect aggression at weaning in pigs. Appl. Anim. Behav. Sci. 
133, 144-153. doi:10.1016/j.applanim.2011.05.018

Miranda-de la Lama, G.C., Sepúlveda, W.S., Montaldo, H.H., María, G.A., Galindo, F., 2011. Social strategies associated with identity profiles in dairy goats. Appl. Anim. Behav. Sci. 134, 48-55. doi:10.1016/j.applanim.2011.06.004

Morton, F.B., Lee, P.C., Buchanan-Smith, H.M., 2013. Taking personality selection bias seriously in animal cognition research: a case study in capuchin monkeys (Sapajus apella). Anim. Cogn. 16, 677-684. doi:10.1007/s10071-013-0603-5

Morton, F.B., Weiss, A., Buchanan-Smith, H.M., Lee, P.C., 2015. Capuchin monkeys with similar personalities have higher-quality relationships independent of age, sex, kinship and rank. Anim. Behav. 105, 163-171. doi:10.1016/j.anbehav.2015.04.013

Näslund, J., Bererhi, B., Johnsson, J.I., 2015. Design of emergence test arenas can affect the results of boldness assays. Ethology $121,556-565$.

Nawroth, C., von Borell, E., Langbein, J., 2015. Object permanence in the dwarf goat (Capra aegagrus hircus): Perseveration errors and the tracking of complex movements of hidden objects. Appl. Anim. Behav. Sci. 167, 20-26. doi:10.1016/j.applanim.2015.03.010

Pike, T.W., Samanta, M., Lindström, J., Royle, N.J., 2008. Behavioural phenotype affects social interactions in an animal network. Proc. R. Soc. Lon. B 275, 2515-2520.

541 Réale, D., Reader, S.M., Sol, D., McDougall, P.T., Dingemanse, N.J., 2007. Integrating animal temperament within ecology and evolution. Biol. Rev. 82, 291-318. doi:10.1111/j.1469-

544 Riemer, S., Müller, C., Virányi, Z., Huber, L., Range, F., 2013. Choice of conflict resolution

Rodríguez-Prieto, I., Martín, J., Fernández-Juricic, E., 2011. Individual variation in behavioural toward an experimenter affects participation, but not performance, in cognitive tests with common marmosets (Callithrix jacchus). Anim. Cogn. 18, 701-712. doi:10.1007/s10071- 
554 Shettleworth, S.J., 2010. Getting Around: Spatial Cognition, in: Cognition, Evolution, and $555 \quad$ Behavior. Oxford University Press, Oxford, pp. 261-312.

556 Sih, A., Bell, A., Johnson, J.C., 2004. Behavioral syndromes: an ecological and evolutionary 557 overview. Trends Ecol. Evol. 19, 372-378.

558 Verbeek, M.E.M., Boon, A., Drent, P.J., 1996. Exploration, aggressive behaviour and 559 dominance in pair-wise confrontations of juvenile male great tits. Behaviour 133, 945-963.

560 Walf, A.A., Frye, C.A., 2007. The use of the elevated plus maze as an assay of anxiety-related 561 behavior in rodents. Nat. Protoc. 2, 322-328.

562 Wolf, M., Van Doorn, G.S., Leimar, O., Weissing, F.J., 2007. Life-history trade-offs favour the 563 evolution of animal personalities. Nature 447, 581-584.

564 Zwick, W.R., Velicer, W.F., 1986. Comparison of five rules for determining the number of 565 components to retain. Psychol. Bull. 99, 432. 
567

568

569

570

571 We assessed exploration (i.e. reaction to a new situations, Réale et al., 2007) using a novel object test (Lansade et al., 2008a) and an altered environment test (Dingemanse et al., 2007),

573 both in familiar environments. Sociability was measured using an attraction test in a familiar environment and an isolation test in a less familiar environment (Lansade et al., 2008b). Tests were conducted during May 2015 and repeated in June 2015. Several behavioural responses

591 Goats were habituated to Pen A over 3 days, for 10 min each day (total: 3 days for 16 goats), during which access to hay and water was given ad libitum. All goats had access to all pens and were measured from each test (Table A.1).

The novel object, altered environment and attraction tests were carried out in a familiar stable complex in a $4.5 \mathrm{~m}$ by $3 \mathrm{~m}$ pen (A), inside the main building of the sanctuary. Pen A was split into quadrants Q1 to Q6 each $1.5 \mathrm{~m} \times 1.5 \mathrm{~m}$ in size (Figure A.1). Because goats were not able to leave the pen during each test, the start quadrant (situated next to the pen gate and away from the test area) was provided as a refuge so that goats could retreat from the test if necessary, in addition to avoiding measuring behaviours unrelated to the exploration personality trait, such as fear and anxiety (Carter et al., 2013; Näslund et al., 2015).

The isolation test was carried out in a separate pen, which was $2 \mathrm{~m}$ by $2 \mathrm{~m}$. The isolation pen was a familiar area, but the subjects were not habituated to it in order to measure behaviours associated with separation anxiety (Réale et al., 2007). This pen was situated at the back of the building to reduce additional stress caused by external factors such as noise or movement. outbuildings at the study site throughout the day. However it was necessary to habituate each goat so that they became accustomed to being handled (Walf and Frye, 2007). In addition, 
595 habituation of goats within Pen A was necessary to reduce anxiety and stress caused by 596 isolation (Cohen and Wills, 1985).

597

598 Individual goats entered the pens through the gate, after which the behaviour of each focal 599 individual was recorded for 5 min with a video camera (Sony HDR-CX190). Recordings started 600 from the point that the experimenter closed the pen gate. After the 5-minute period, the pen 601 gate was opened and the focal goat was free to leave the test pen. Each individual test was 602 carried out on the same day, and repeated 3 weeks after the initial test had taken place using 603 the same methodology. The order in which goats were tested was random on each test day, 604 and for each Repeat.

605 
a)

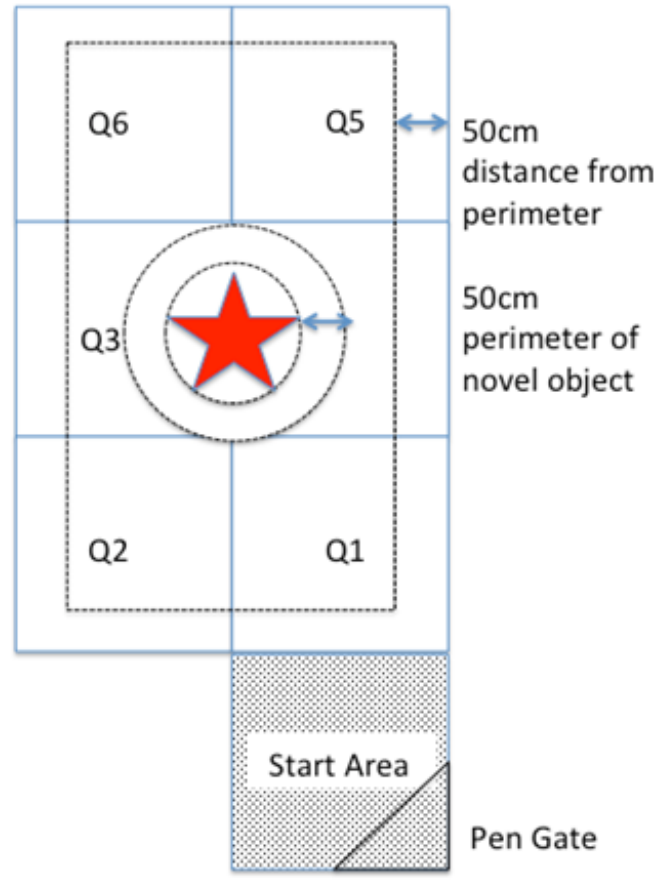

c)

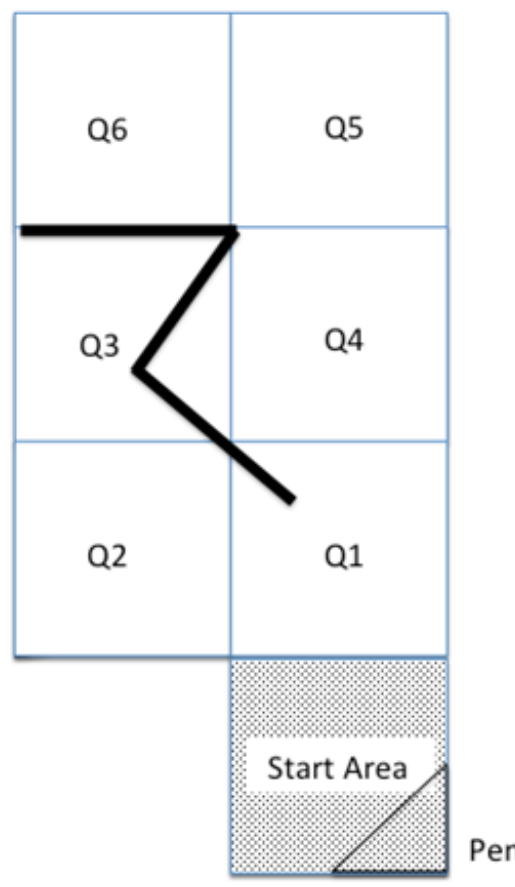

b)

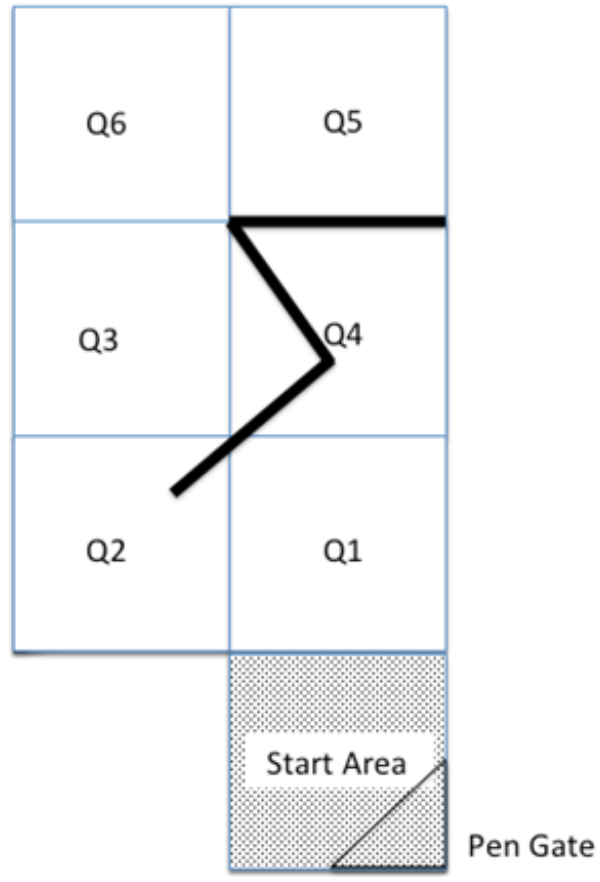

d)

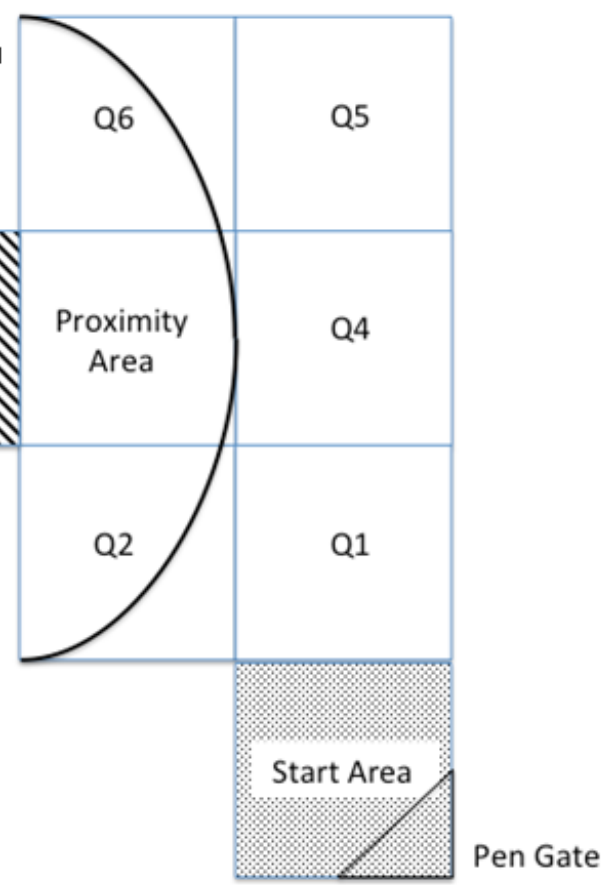

607 Figure A1. Test setups for the a) novel object test b) and c) altered environment test d) attraction test 
610 Exploration

611

612 The novel object test was carried out in Pen A with a novel item, which was placed on the floor 613 in the middle of quadrant 3 and 4 (Figure A.1a). In the first Repeat, the novel object was an 614 inflatable child's toy that was $40 \mathrm{~cm}$ high and $30 \mathrm{~cm}$ in length. The second Repeat used a large 615 multi-coloured beach ball that was $40 \mathrm{~cm}$ high. The altered environment test was also carried 616 out inside Pen A. The layout of the pen was altered from the normal layout to which goats were 617 habituated to in the habituation phase. Gates were used to create an altered environment in two 618 different layouts in the first and second Repeat of this test (Figure A.1b-c).

619

620 Sociability

621

622 The attraction test was carried out in Pen A, with the pen mate of the focal goat housed in an 623 adjacent pen B, where focal goats could easily view and touch pen mates (Figure A.1d). Once 624 the pen mate was housed inside the adjacent pen, the focal goat then entered Pen A through 625 the gate by the start quadrant after which recording of the focal goat began. The isolation test 626 was carried out in a separate pen. Individual goats entered the pen and the gate was closed 627 behind them. The behaviour of each individual goat was recorded, starting from the point that 628 the experimenter left the area and was out of sight of the focal goat. After the 5-min test period, 629 the focal goat was released and free to leave the test pen.

630

631 
633 Adapted from Briefer et al. (2015).




637 Behaviours recorded during each test were scored using CowLog 2.0 (Hänninen and Pastell, 2009). A template outlining each quadrant, in addition to proximities from pen walls, novel objects and pen mates, was placed over the computer screen when viewing videos for consistency of scoring proximity measures. Table A.1 shows the ethogram used for each personality test, and outlines all behaviours recorded along with their descriptions.

642

A principal component analysis (PCA) was carried out in order to combine all behavioural parameters in one score for each personality trait, which would be representative of the response of individuals to the personality tests (McGregor, 2013). All the behavioural data taken during the novel object and altered environment tests from the first Repeat were included together in one PCA. A second PCA was calculated using the data collected from the second Repeat. This resulted in two PCA results, one each for Repeat 1 and 2 for the exploration trait. This process was then repeated using the behavioural data taken during the isolation and attraction tests and resulted in a further two PCA results, one for each Repeat 1 and 2 for the sociability trait.

652

653 The PCA output provided the loadings of each behaviour on each principal component. The loadings reflect the weight of each behaviour within the component. Only loadings of $\leq-0.3$ or $\geq$ 0.3 were used for reliable PC interpretation (Comrey and Lee, 2013) in addition to eigenvalues $\geq 1.0$ for each principle component (Zwick and Velicer, 1986). The first four PCs from the PCA analysis for exploration and the first five PCs for sociability were used in the final analysis as these PC's had eigenvalues $\geq 1.0$. Loadings from PC1 for both exploration and sociability values were representative of each trait and were therefore used to calculate personality scores for each trait. The data from PC1 for Repeat 1 and 2 of each personality traits was tested for normality using an Anderson Darling test (Exploration (Repeat 1), $A D=0.25, \mathrm{n}=16$ goats, $P=0.72$; (Repeat 2$) \mathrm{AD}=0.34, \mathrm{n}=16$ 
goats, $P=0.46$; Sociability (Repeat 1 ), $A D=0.33, \mathrm{n}=16$ goats, $P=0.46$; (Repeat 2 ) $A D=0.49$ $\mathrm{n}=16$ goats, $P=0.19)$. As all data was normally distributed, parametric correlations were calculated to check for consistency between the repeats of each personality traits. (Pearson's Correlation: Exploration $r_{\mathrm{p}}=0.826, \mathrm{n}=16$ goats, $P<0.001$; Sociability $r_{\mathrm{p}}=0.83, \mathrm{n}=16$ goats, $P$ $<0.001)$. Values were consistent between repeats for both exploration and sociability. Means of Repeat 1 and 2 for each personality trait were then calculated to obtain personality scores for each individual goat. All statistical analyses were performed using Minitab (Release 13.1 ( ) 2000 Minitab Inc.).

672

Results

674

The output from the PCA performed on the exploration data for Repeat 1 and 2 revealed 4 PCs with an eigenvalue greater than 1 (Table A.2). These 4 PCs for Repeat 1 and 2 explained 81.0 $\%$ and $82.6 \%$ of the variance respectively. According to the loadings of the behaviours on the PCs (Table A.2), the first PC (PC1) reflected exploration behaviour well across both tests for each Repeat (novel object and altered environment; i.e. goats that were highly explorative in both tests). The following behaviours were correlated with the first PC for both Repeats (loadings $\leq-0.3$ or $\geq 0.3$ ): time spent immobile exploring (for altered environment), time spent in start quadrant and frequency of quadrant entered (Table A.2). Highly positive PC1 values indicated goats that were explorative and spent time investigating the novel object or altered environment. Highly negative PC1 values indicated goats that did not investigate the novel object or environment (i.e. spent the majority of time in the start area or at the perimeter of the pen). The duration of exploring the novel object or environment was shorter in these goats, compared to subjects with higher exploration scores. These goats did not explore the object or altered environment at all.

689

690 The output from the PCA performed on the sociability data for Repeat 1 and 2 revealed 5 PCs 691 with an eigenvalue greater than 1 (Table A.2). These 5 PCs explained $83.0 \%$ and $80.9 \%$ of the variance respectively, for each Repeat 1 and 2 . According to the loadings of the behaviours on 
693 the PCs (Table A.2), the first PC (PC1) reflected sociability well across both tests for each

694 Repeat (attraction and isolation; i.e. goats that were showing increased social responses in both 695 tests, spending time touching companions during the attraction test and reacting strongly to 696 isolation). The following behaviours were correlated with the first PC for both Repeats (loadings $697 \leq-0.3$ or $\geq 0.3$ ): time contact companion (for attraction test), transitional behaviour (for both 698 isolation and attraction tests) and call frequency (for attraction test). Highly positive PC1 values 699 indicated goats that were sociable and spent time in contact with companions. Goats with a high 700 sociability score exhibited a reduction in time spent feeding (or did not feed at all), had higher 701 rates of locomotion within the pen and an increased call frequency during the isolation test, 702 compared to goats with lower sociability scores (Table A.2). 
Table A2. Factor loadings of the measured behavioural parameters for the principal components for test on exploration and sociability (Repeat 1 and

\begin{tabular}{|c|c|c|c|c|c|c|c|c|c|c|c|c|c|c|}
\hline \multirow[b]{2}{*}{ Trait } & \multirow[b]{2}{*}{ Test } & \multirow[b]{2}{*}{ Behaviour } & & & & & & & & & & & & \\
\hline & & & \multirow{30}{*}{ 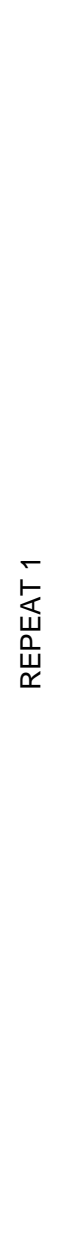 } & PC1 & PC2 & PC3 & PC4 & PC5 & \multirow{30}{*}{ 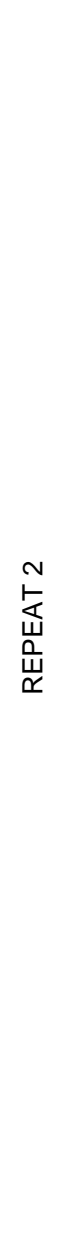 } & PC1 & PC2 & PC3 & PC4 & PC5 \\
\hline \multirow{15}{*}{$\begin{array}{l}\text { 을 } \\
\frac{\pi}{\overline{0}} \\
\frac{0}{2} \\
\text { × }\end{array}$} & \multirow{5}{*}{ Novel Object } & Time Interact & & 0.222 & 0.01 & -0.543 & -0.134 & & & 0.32 & 0.185 & 0.035 & -0.291 & \\
\hline & & Latency Interact Object & & -0.221 & -0.332 & 0.116 & 0.095 & & & -0.136 & -0.11 & 0.46 & 0.357 & \\
\hline & & Time in Object Proximity & & 0.239 & 0.167 & -0.439 & 0.057 & & & 0.288 & -0.106 & -0.332 & -0.076 & \\
\hline & & Time Start Quadrant & & -0.303 & -0.354 & -0.005 & 0.373 & & & -0.257 & 0.4 & 0.276 & 0.232 & \\
\hline & & Time Perimetre & & 0.163 & 0.295 & 0.366 & -0.45 & & & 0.059 & -0.528 & -0.035 & -0.288 & \\
\hline & \multirow{8}{*}{ Altered Environment } & Latency to Explore & & -0.308 & 0.16 & -0.171 & -0.451 & & & 0.27 & 0.331 & -0.053 & 0.032 & \\
\hline & & Time Mobile Explore & & 0.278 & -0.205 & -0.252 & 0.075 & & & 0.343 & 0.271 & -0.014 & 0.199 & \\
\hline & & Time Immobile Explore & & 0.314 & -0.394 & -0.116 & -0.057 & & & -0.379 & 0.172 & 0.049 & -0.253 & \\
\hline & & Time Start Quadrant & & -0.366 & -0.144 & -0.291 & -0.298 & & & 0.379 & -0.172 & -0.049 & 0.253 & \\
\hline & & Time Quadrant 1-6 & & 0.366 & 0.144 & 0.291 & 0.298 & & & 0.021 & -0.44 & 0.43 & 0.021 & \\
\hline & & Quadrant Frequency & & 0.357 & -0.068 & 0.033 & -0.217 & & & 0.338 & -0.114 & 0.291 & 0.336 & \\
\hline & & Locomotion & & 0.014 & -0.392 & 0.29 & -0.375 & & & -0.341 & -0.219 & -0.309 & 0.197 & \\
\hline & & Time Standing & & -0.244 & 0.477 & -0.063 & 0.222 & & & 0.133 & 0.082 & 0.476 & -0.569 & \\
\hline & & Eigenvalue & & 4.3209 & 2.8317 & 2.1453 & 1.2324 & & & 5.4373 & 2.2518 & 1.7786 & 1.273 & \\
\hline & & Cum \% variance & & 33.2 & 55 & 71.5 & 81 & & & 41.8 & 59.1 & 72.8 & 82.6 & \\
\hline \multirow{14}{*}{$\begin{array}{l}\text { त्र } \\
\frac{\overrightarrow{0}}{\overline{0}} \\
\frac{\pi}{0} \\
\dot{\infty}\end{array}$} & \multirow{6}{*}{ Attraction } & Time Companion Proximty & & -0.033 & 0.321 & 0.431 & 0.257 & 0.224 & & 0.22 & 0.053 & -0.219 & -0.089 & 0.682 \\
\hline & & Latency to Companion & & 0.078 & -0.27 & -0.08 & -0.275 & -0.599 & & -0.212 & -0.418 & 0.164 & 0.014 & -0.421 \\
\hline & & Calls & & 0.027 & 0.443 & -0.406 & -0.177 & -0.008 & & 0.343 & 0.036 & 0.229 & 0.219 & -0.209 \\
\hline & & Time Contact Companion & & 0.349 & -0.301 & -0.106 & 0.041 & 0.013 & & 0.326 & -0.053 & 0.241 & 0.031 & 0.158 \\
\hline & & Locomotion & & 0.097 & 0.511 & 0.061 & 0.267 & -0.209 & & 0.228 & 0.417 & 0.015 & 0.223 & -0.147 \\
\hline & & Transitional Behaviour & & 0.351 & 0.179 & -0.064 & 0.172 & -0.32 & & 0.35 & 0.118 & 0.106 & 0.289 & -0.086 \\
\hline & \multirow{6}{*}{ Isolation } & Latency Feed & & 0.203 & 0.08 & 0.453 & -0.385 & 0.006 & & 0.207 & 0.068 & -0.371 & -0.147 & -0.356 \\
\hline & & Time Feed & & -0.172 & -0.072 & -0.488 & 0.101 & 0.44 & & -0.226 & -0.207 & 0.371 & 0.025 & 0.108 \\
\hline & & Locomotion & & 0.127 & 0.433 & -0.261 & -0.319 & -0.057 & & 0.19 & 0.315 & 0.128 & -0.542 & -0.204 \\
\hline & & Time Lying or Kneeling & & -0.188 & -0.063 & -0.192 & 0.458 & -0.404 & & -0.049 & 0.094 & -0.398 & 0.514 & -0.105 \\
\hline & & Transitional Behaviour & & 0.324 & -0.02 & -0.241 & -0.232 & 0.142 & & 0.328 & -0.058 & 0.094 & -0.352 & -0.145 \\
\hline & & Calls & & 0.362 & -0.129 & 0.056 & -0.062 & 0.205 & & 0.276 & -0.28 & 0.164 & 0.033 & 0.196 \\
\hline & & Eigenvalue & & 5.0437 & 2.6637 & 1.9349 & 1.7387 & 1.1301 & & 5.9695 & 1.9717 & 1.8558 & 1.2615 & 1.0773 \\
\hline & & Cum \% variance & & 33.6 & 51.4 & 64.3 & 75.9 & 0.834 & & 39.8 & 52.9 & 65.3 & 73.7 & 0.809 \\
\hline
\end{tabular}


